\title{
Flight of fancy
}

\section{How an 'eccentric' line of research proved its worth.}

\section{Carol Robinson}

rom an early age I have been fascinated by the workings of a mass spectrometer. At the age of 16, working in a drug discovery laboratory, I would spend many hours optimizing the mass spectra of small organic chemicals. These volatile molecules were easily vaporized and transmitted into the gas phase, allowing their masses to be determined with high accuracy.

At first I was satisfied with my role as technician, but quickly became frustrated by my lack of scientific background. After many years of part-time study, I graduated in chemistry and later received a $\mathrm{PhD}$ in mass spectrometry from Cambridge University. This training stood mein good stead for the developments that took place during my career break. After almost a decade away from science, spent raising my three children, I returned to mass spectrometry and found the same fascination remained. But the mass scale at which I now operated had increased an order of magnitude. During my absence, a momentous innovation had taken place - that of electrospray mass spectrometry. This revolutionary process produced a fine spray from a solution of biomolecules and removed the requirement for volatility. Because of this, it became possible to spray intact proteins into the gas phase of the spectrometer, paving the way for the widespread applications in proteomics that quickly followed this discovery.

I was fortunate to have the opportunity to work with this new technique in the chemistry department at Oxford University. At that time (the early 1990s), the department was using electrospray to characterize small molecules and denatured proteins. I negotiated one day a week on the spectrometer, to indulge in what could be considered quite obscure areas of research. I especially wanted to introduce proteins in conditions where we could maintain their native state in the electrospray droplet, so that I could study the folding process. In the course of this research, I noticed that cofactors and other proteins would sometimes adhere to the folding protein molecule. I became interested in trying to understand this phenomenon, as it had always been assumed that weak interactions between proteins and cofactors would not survive the transition from solution to gas phase.

Despite several early setbacks, I eventually found conditions whereby I could maintain folding and interactions. But my early efforts were restricted by mass range, to complexes containing no more than three or

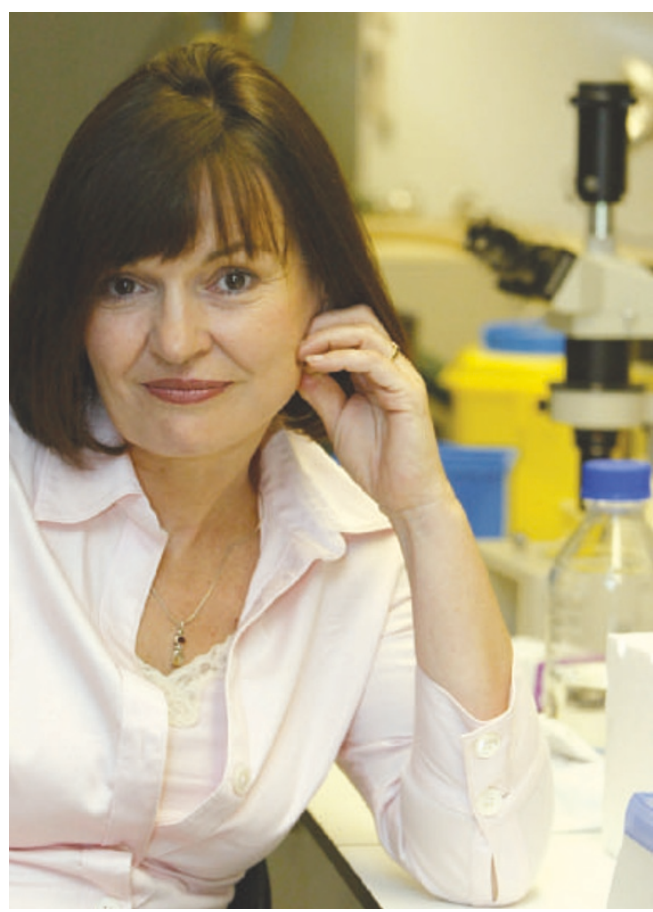

Critical mass: Carol Robinson pushes the limits of a mass spectrometer to characterize large protein complexes.

four proteins. So I was enthusiastic when invited to look at a prototype mass spectrometer that had an electrospray source interfaced with a time-of-flight detector. I hoped that this device would give me the unlimited mass range I wanted and enable me to look at significantly larger protein complexes. I took some of my most challenging complexes with me, determined to put this spectrometer through its paces and make it work for me.

Unfortunately, my proposal was not greeted with enthusiasm. The instrument had been optimized for high-precision applications, specifically for sequencing peptides. I was seen to want to destroy all the benefits that had been introduced, reducing performance for the sake of obscure research. After several fruitless attempts in which my complexes fell apart into their components, even I was beginning to wonder if this was worth doing. Late one evening, in a last-ditch attempt, we went against traditional mass spectrometry folklore and actively impaired the vacuum system. This action led to success! We were able to observe ions from an assembly of 14 protein subunits of the heat-shock protein GroEL. With a molecular mass of 800,000 daltons, this was the largest protein assembly (at that time) to yield a mass spectrum. Although it was already known that GroEL had 14 subunits, this one spectrum represented a turning point for me and held the promise of many opportunities.
Encouraged by this success and with the beginnings of a research team, we attempted to push the mass range further by projecting into the mass spectrometer intact ribosomes and viruses with masses greater that 2 million daltons, as well as other molecular chaperones. Although the mass spectra of viruses were accepted conceptually (it is well established that their transmission into the atmosphere is all too easy), projecting intact ribosomes into the mass spectrometer was considered more than a little eccentric! I remember, on more than one occasion, being teased by colleagues with suggestions that I was on the 'lunatic fringe'. I was also frequently challenged at conferences. It would be pointed out that the structures of large assemblies in the gas phase were unknown, their formation not understood, and consequently my research was of little significance. Moreover, at that time it was not clear - even to me - what these experiments would show. Examining relatively small molecules provides a unique and identifiable property - that of mass and sometimes molecular composition. For assemblies such as the ribosome, containing upwards of 200,000 atoms, molecular mass is not very informative. We had to find ways of making our unique spectra work for us.

It quickly became apparent that heterogeneity and dynamics were two properties on which we had a new handle. We could measure in real time changes in masses of complexes as they assembled from their components, exchanged subunits between assemblies and changed their folding in response to the addition of factors. These attributes gave us unique insight into protein assemblies that refuse to conform to the requirement of X-ray crystallographers to exist in one rigid form. When working with ribosomes, we discovered that we could examine the dynamic complex known as the'stalk', which acts like an independent arm in delivering factors to the ribosome. Interestingly - even in high-resolution structures - no individual proteins can be discerned for this part of the ribosome. We were able to uncover both dynamic changes in response to factor binding and a speciesdependent stoichiometry.

This short and personal account highlights an interesting aspect of scientific investigation: that the distinction between 'eccentric' and 'progressive' can only be made in hindsight.

Carol Robinson is in the Department of Chemistry, University of Cambridge, Lensfield Road,

Cambridge CB2 1EW, UK. 